Original

\title{
Valor pronóstico de la desnutrición en pacientes con insuficiencia cardíaca aguda y su influencia en la interpretación de marcadores de congestión venosa sistémica
} \author{
y Juan Ignacio Pérez-Calvo a,b,c \\ a Servicio de Medicina Interna, Hospital Clínico Universitario «Lozano Blesa», Zaragoza, España \\ ${ }^{\mathrm{b}}$ Instituto de Investigación Sanitaria de Aragón (IIS), Zaragoza, España \\ ${ }^{c}$ Facultad de Medicina, Universidad de Zaragoza, Zaragoza, España \\ ${ }^{\mathrm{d}}$ Instituto Aragonés de Ciencias de la Salud, Zaragoza, España \\ e Servicio de Medicina Interna, Hospital Universitario Arnau de Villanova, Lérida, España
}

Jorge Rubio-Gracia ${ }^{\mathrm{a}, \mathrm{b}, *}$, Claudia Josa-Laorden ${ }^{\mathrm{a}, \mathrm{b}}$, Marta Sánchez-Marteles ${ }^{\mathrm{a}, \mathrm{b}}$, Ignacio Giménez-López ${ }^{\mathrm{b}, \mathrm{c}, \mathrm{d}}$, Vanesa Garcés Horna ${ }^{\mathrm{a}, \mathrm{b}}$, José Luis Morales Rulle

\section{INFORMACIÓN DEL ARTÍCULO}

\section{Historia del artículo:}

Recibido el 28 de abril de 2020

Aceptado el 24 de junio de 2020

On-line el xxx

\section{Palabras clave:}

Insuficiencia cardíaca

Desnutrición

Síndrome cardio-renal

Congestión

\begin{abstract}
R E S U M E N
Antecedentes: La desnutrición es frecuente en los pacientes con insuficiencia cardíaca(IC). Dicha situación contribuye al incremento de la congestión sistémica dificultando el manejo clínico. Cuantificar la desnutrición y su relación con la congestión sistémica, es importante para optimizar el tratamiento durante la fase aguda.

Material y métodos: Estudio de cohortes retrospectivo en pacientes con diagnóstico de IC aguda. La población se estratificó según los índices de nutrición de CONUT (Controlling Nutritional Status) y PNI (Prognostic Nutritional Index) con el objetivo de analizar su relación con parámetros objetivos de congestión y el valor pronóstico al año de seguimiento.

Resultados: Se incluyeron un total de 309 pacientes, presentando más de la mitad algún grado de desnutrición al ingreso. El grado de congestión fue significativamente superior en los pacientes desnutridos, con una mayor proporción de «líneas b» y un mayor volumen plasmático relativo. Las concentraciones de la prohormona N-terminal del péptido natriurético cerebral (NT-proBNP), tanto al ingreso como al alta, también fueron significativamente superiores en los pacientes desnutridos, independientemente de la escala empleada. El análisis univariante identificó el índice de CONUT y PNI, como factores asociados a la mortalidad al año para todas las causas (HR 1,62 [1,22-2,14]; $\mathrm{p}=0,001)$ y de PNI (HR 0,65 [0,53-0,80]; $\mathrm{p}=<0,001)$, respectivamente.

Conclusiones: Un mayor grado de desnutrición (determinado mediante los índices de CONUT y PNI) en pacientes con IC aguda, se asoció a una mayor presencia de parámetros objetivos de congestión y a una mayor mortalidad al año para todas las causas.
\end{abstract}

(C) 2020 Elsevier España, S.L.U. Todos los derechos reservados.

\section{Prognostic value of malnutrition in patients with acute heart failure and its influence on the interpretation of markers of systemic venous congestion}

\section{A B S T R A C T}

Background: Malnutrition is frequent in patients with heart failure (HF) and contributes to increased systemic congestion, but also hinders its correct assessment, especially during decompensations. Estimating the degree of malnutrition and its relationship with systemic congestion is important to optimize treatment during decompensations.

\footnotetext{
* Autor para correspondencia.

Correo electrónico: jorgerubiogracia@gmail.com (J. Rubio-Gracia).
} 
Material and methods: Retrospective cohort study in patients with acute HF. The population was stratified according to CONUT (Controlling Nutritional Status) and PNI (Prognostic Nutritional Index) nutrition indices in order to analyse their relationship with objective parameters of congestion and the prognostic value of malnutrition.

Results: 309 patients were included. More than half presented some degree of malnutrition upon admission. The degree of congestion was significantly higher in malnourished patients, with a higher proportion of "comet tail artifacts" and a higher relative plasma volume. NT-proBNP concentrations, both on admission and at discharge, were also significantly higher in malnourished patients, regardless of the scale used. The univariate analysis identified the CONUT and PNI index as factors associated with one-year mortality from any cause (HR 1.62 [1.22-2.14]; $\mathrm{p}=0.001$ ) and PNI (HR 65 [0.53-0.80]; $\mathrm{p}=<0.001$ ), respectively. Conclusions: A higher degree of malnutrition (determined by means of the CONUT and PNI indices) in patients with acute HF was associated with a higher presence of objective parameters of congestion and a higher one-year all-cause mortality.

(c) 2020 Elsevier España, S.L.U. All rights reserved.

\section{Introducción}

La insuficiencia cardíaca (IC), es una causa frecuente de ingreso en los servicios de Medicina Interna ${ }^{1}$. Se calcula que aproximadamente el 70\% de los pacientes presentan antecedentes de IC crónica, síndrome asociado con una alta carga de comorbilidades ${ }^{2}$, entre las que se encuentra la desnutrición ${ }^{2}$.

Según la herramienta empleada en su estimación, hasta la mitad de los pacientes ingresados por IC, presentan algún grado de desnutrición ${ }^{3}$. Su incidencia es mayor en pacientes de más edad y mayor grado de dependencia ${ }^{4,5}$. La disminución del aporte calóricoproteico en la dieta, junto con el incremento de mediadores inflamatorios, condicionan la aparición de una pérdida progresiva de masa muscular o sarcopenia, que en casos extremos da lugar a la denominada "caquexia cardíaca»" ${ }^{3}$, caracterizada por anorexia, edemas y pérdida marcada de la masa muscular.

La desnutrición confiere un peor pronóstico a los pacientes con IC. Múltiples estudios relacionan los diferentes grados de desnutrición con el pronóstico en términos de mortalidad y/o reingresos por IC. La estimación del grado de desnutrición puede hacerse de varios modos, ya sea mediante parámetros objetivos como la pérdida de peso o el índice de masa corporal (IMC) o mediante escalas validadas como el índice de CONUT $^{6}$ (Controlling Nutritional Status), el GNRI ${ }^{7}$ (Geriatric Risk Index) o el PNI (Prognostic Nutritional Index $)^{8}$.

Por otro lado, la mayoría de los pacientes que ingresan por IC aguda presentan signos y/o síntomas de congestión ${ }^{9}$, que pueden verse modificados por el estado nutricional de los pacientes, incrementando, por ejemplo, los edemas en porciones declives y dificultando la interpretación de los hallazgos en la exploración física ${ }^{10,11}$. De hecho, esta situación ha facilitado el avance en la búsqueda de marcadores de congestión objetivos, que no se vean influenciados por la desnutrición, a fin de poder cuantificar de una manera más precisa, el grado de congestión y poder guiar el tratamiento diurético intravenoso de una manera más efectiva ${ }^{12}$.

Por lo tanto, conocer el estado nutricional de los pacientes con IC y su relación con la congestión venosa sistémica, ya sea mediante la exploración física ${ }^{13}$ o el uso de marcadores de congestión ${ }^{14}$, es fundamental para optimizar el tratamiento descongestivo durante la fase de hospitalización y mejorar el pronóstico de los mismos ${ }^{15}$.

Los objetivos de este estudio fueron: 1) Analizar el estado nutricional de los pacientes con IC descompensada ingresados en Medicina Interna, mediante las escalas nutricionales de CONUT y PNI; 2) Analizar el valor pronóstico de la desnutrición para el objetivo de muerte por todas las causas al año de la hospitalización; 3) Analizar la relación existente entre la desnutrición (escalas de CONUT y PNI) y los marcadores de congestión.

\section{Pacientes y métodos}

\section{Población a estudio}

Estudio de cohortes retrospectivo llevado a cabo en el Hospital Clínico Universitario «Lozano Blesa» de Zaragoza, España, entre los años 2013 y 2018. Todos los pacientes incluidos ingresaron en el servicio de Medicina Interna con el diagnóstico de insuficiencia cardíaca aguda o descompensada y recibieron posteriormente seguimiento en una consulta monográfica de IC durante, al menos, un año. El seguimiento (número y frecuencia de las visitas) y tratamiento de los pacientes fue realizado según el criterio de los facultativos responsables de cada paciente.

Los criterios de inclusión fueron: 1) Edad mayor de 18 años con diagnóstico de IC aguda (ya fuera de novo, o reagudización de una insuficiencia cardíaca crónica) al ingreso; 2) Concentraciones basales de NT-proBNP $\geq 750 \mathrm{pg} / \mathrm{mL}$ (punto de corte establecido por el laboratorio de referencia del centro); 3) Firma de consentimiento informado para el almacenamiento de muestras biológicas (en caso de que se solicitaran), y 4) Aceptación de seguimiento en las consultas externas de IC. Los criterios de exclusión fueron: 1) Estancia previa en el ingreso índice en la Unidad de Cuidados Intensivos; 2) Necesidad de tratamiento renal sustitutivo; 3) Enfermedad valvular significativa (estenosis aórtica severa, estenosis mitral severa o insuficiencia mitral severa); 4) Enfermedad pulmonar obstructiva crónica (EPOC) avanzada (definida por volumen espiratorio forzado en el primer segundo [FEV1] < 30\%) e 5) IC aguda secundaria a la presencia de arritmias (a excepción de la fibrilación auricular/flutter rápida).

En todos los pacientes se realizó una ecocardiografía 2D durante los seis meses previos a su inclusión en el estudio o en el mes posterior al alta. La fracción de eyección ventricular izquierda (FEVI) fue calculada según las guías de imagen ecocardiográficas vigentes durante el periodo del estudio ${ }^{16}$.

El estudio cumplió con las directrices fundamentales de la Declaración Internacional de Helsinki. Aquellos pacientes en los que se requirió alguna muestra adicional de sangre, firmaron un consentimiento informado para su extracción y almacenamiento a $-80^{\circ} \mathrm{C}$. Las muestras así obtenidas se amparan en el registro BIOMIC (Inscrito en el Registro Nacional de Biobancos [sección colecciones], código de referencia C.0000400 con fecha 5 de octubre de 2012 y fue aprobado por el CEICA, Ref. C.P.-C.I. PI12/00117, 3 de octubre de 2012).

\section{Análisis del estado nutricional}

El estado nutricional de los pacientes se analizó mediante el uso de diferentes parámetros: 1) Índice de CONUT $^{6}$, calculado a 
Tabla 1

Características basales según el índice de CONUT al ingreso

\begin{tabular}{|c|c|c|c|c|c|}
\hline Variables & Normal & Leve & Moderado & Severo & p-valor \\
\hline$N(\%)$ & $13(4,2)$ & $89(28,8)$ & $170(55,0)$ & $37(12,0)$ & \\
\hline Edad (años) & $79,6(75,7-84,0)$ & $80,6(73,2-94,6)$ & $83,0(78,7-87,3)$ & $84,0(78,3-89,0)$ & 0,003 \\
\hline Género: (hombres[\%]) & $5(38,5)$ & $42(47,2)$ & $82(48,2)$ & $24(64,9)$ & 0,219 \\
\hline Peso $(\mathrm{kg})$ & $68,5(63,7-81,0)$ & $76,0(65,0-92,5)$ & $73,5(64,7-82,2)$ & $74,0(64,5-85,5)$ & 0,611 \\
\hline $\operatorname{Altura}(m)$ & $1,62(1,51-1,70)$ & $1,59(1,52-1,65)$ & $1,60(1,53-1,68)$ & $1,62(1,54-1,73)$ & 0,436 \\
\hline$I M C\left(\mathrm{~kg} / \mathrm{m}^{2}\right)$ & $27,8(23,9-31,2)$ & $30,8(25,9-34,7)$ & $28,3(25,2-33,0)$ & $27,0(25,1-31,6)$ & 0,141 \\
\hline $\mathrm{TAS}(\mathrm{mmHg})$ & $150(128-185)$ & $135(124-160)$ & $132(117-150)$ & $130(118-148)$ & 0,032 \\
\hline$T A D(m m H g)$ & $77(67-87)$ & $76(67-89)$ & $70(62-81)$ & $70(58-78)$ & 0,018 \\
\hline$F C(l / p / m)$ & $80(74-98)$ & $79(66-96)$ & $77(65-92)$ & $80(73-93)$ & 0,358 \\
\hline \multicolumn{6}{|l|}{ Signos de IC ( $n[\%])$} \\
\hline IY & $7(53,8)$ & $43(49,4)$ & $114(68,7)$ & $24(68,6)$ & 0,020 \\
\hline Edemas & $8(61,5)$ & $71(81,6)$ & $132(79,0)$ & $31(88,6)$ & 0,220 \\
\hline Crepitantes & $9(81,8)$ & $48(64,0)$ & $109(76,8)$ & $20(66,7)$ & 0,179 \\
\hline \multicolumn{6}{|l|}{ Parámetros ecocardiográficos } \\
\hline FEVI $(\%)$ & $63,5(32,5-65,0)$ & $50,5(40,0-61,5)$ & $54,5(42,5-64,5)$ & $60,5(46,2-65,0)$ & 0,367 \\
\hline $\mathrm{PAP}(\mathrm{mmHg})$ & $41(24-48)$ & $48(32-55)$ & $45(35-59)$ & $43(37-60)$ & 0,893 \\
\hline Diámetro AI (mm) & $44,9(5,8)$ & $47,5(6,9)$ & $49,1(10,1)$ & $50,1(10,8)$ & 0,295 \\
\hline Masa ventricular $(\mathrm{g})$ & $119(22)$ & $120(37)$ & $124(53)$ & $126(49)$ & 0,949 \\
\hline «Líneas b» ecográficas (presencia) & $5(41,7)$ & $26(40,6)$ & $84(68,9)$ & $14(58,3)$ & $\mathbf{0 , 0 0 2}$ \\
\hline Dilatación VCI ( $\geq 50 \%)$ & $2(28,6)$ & $13(40,6)$ & $36(55,4)$ & $7(58,3)$ & 0,314 \\
\hline Clase funcional basal NYHA (n[\%]) & & & & & 0,453 \\
\hline I & $2(0,7)$ & $14(4,7)$ & $14(4,7)$ & $3(1,0)$ & \\
\hline II & $9(69,2)$ & $55(63,2)$ & $105(64,4)$ & $19(52,8)$ & \\
\hline III & $2(15,4)$ & $18(20,7)$ & $43(26,4)$ & $14(38,9)$ & \\
\hline IV & $0(0,0)$ & $0(0,0)$ & $1(0,6)$ & $0(0,0)$ & \\
\hline \multicolumn{6}{|l|}{ Antecedentes ( $n[\%])$} \\
\hline ICC & $7(53,8)$ & $62(69,7)$ & $118(69,8)$ & $27(73,0)$ & 0,654 \\
\hline Hipertensión & $11(84,6)$ & $71(79,8)$ & $144(84,7)$ & $33(89,2)$ & 0,575 \\
\hline Cardiopatía isquémica & $0(0,0)$ & $32(36,0)$ & $54(31,8)$ & $11(29,7)$ & 0,013 \\
\hline Dislipemia & $5(38,5)$ & $48(53,9)$ & $77(45,6)$ & $11(29,7)$ & 0,089 \\
\hline Diabetes & $7(53,8)$ & $33(37,1)$ & $70(41,2)$ & $16(43,2)$ & 0,676 \\
\hline FA/flutter & $6(46,2)$ & $53(59,6)$ & $105(61,8)$ & $18(48,6)$ & 0,381 \\
\hline EPOC & $2(15,4)$ & $9(10,1)$ & $33(19,4)$ & $6(16,2)$ & 0,264 \\
\hline ERC & $3(23,1)$ & $19(21,3)$ & $60(35,5)$ & $16(43,2)$ & 0,037 \\
\hline Anemia & $3(23,1)$ & $14(15,9)$ & $48(28,4)$ & $12(32,4)$ & 0,096 \\
\hline Portador marcapasos & $4(30,8)$ & $6(6,7)$ & $20(11,8)$ & $3(8,1)$ & 0,110 \\
\hline \multicolumn{6}{|l|}{ Tratamiento IC (n[\%]) } \\
\hline Diuréticos de asa & $12(92,3)$ & $79(88,8)$ & $147(91,3)$ & $28(82,4)$ & 0,507 \\
\hline Betabloqueadores & $7(53,8)$ & $51(57,3)$ & $86(53,4)$ & $13(38,2)$ & 0,302 \\
\hline IECA/ARA II & $11(84,6)$ & $65(73,0)$ & $120(74,5)$ & $18(52,9)$ & 0,064 \\
\hline BRM & $4(30,8)$ & $28(31,5)$ & $45(28,1)$ & $10(30,3)$ & 0,954 \\
\hline Variables & Normal & Leve & Moderado & Severo & p-valor \\
\hline \multicolumn{6}{|l|}{ Biomarcadores } \\
\hline NT-proBNP al ingreso (pg/mL) & $1.176(836-4.034)$ & $2.591(1.611-5.350)$ & $4.278(1.913-7.742)$ & $4.629(2.748-9.106)$ & 0,003 \\
\hline NT-proBNP al alta $(\mathrm{pg} / \mathrm{mL})$ & $1.016(218-1.912)$ & $1.808(756-2.832)$ & $2.757(1.343-5.097)$ & $3.049(1.427-5.260)$ & 0,001 \\
\hline CA125 al ingreso $(\mathrm{mU} / \mathrm{mL})$ & $35,0(21,5-99,0)$ & $46,5(16,8-108,2)$ & $58,3(30,1-113,6)$ & $65,7(34,0-126,2)$ & 0,104 \\
\hline CA125 al alta $(\mathrm{mU} / \mathrm{mL})$ & $41,7(14,5-127,9)$ & $39,0(12,9-109,1)$ & $68,4(32,1-131,3)$ & $97,6(26,3-173,0)$ & 0,022 \\
\hline \multicolumn{6}{|l|}{ Análisis indirecto del volumen plasmático } \\
\hline Volumen plasmático real (mL) & $2.464(2.171-2.941)$ & $2.827(2.437-3.128)$ & $2.875(2.518-3.191)$ & $2.954(2.565-3.285)$ & 0,247 \\
\hline Volumen plasmático ideal (mL) & $2.740(2.486-3.219)$ & $3.003(2.593-3.616)$ & $2.899(2.525-3.247)$ & $2.886(2.547-3.334)$ & 0,571 \\
\hline $\operatorname{VPr}(\%)$ & $-9,2([-17,2]-[-1,5])$ & $-5,7([-14,1]-[0,5])$ & $-2,1([-8,6]-[6,1])$ & $-1,1([-4,9]-[4,6])$ & 0,002 \\
\hline \multicolumn{6}{|l|}{ Parámetros de función renal } \\
\hline Cistatina al ingreso $(\mathrm{mg} / \mathrm{mL})$ & $1,41(1,10-1,78)$ & $1,40(1,12-1,91)$ & $1,45(1,21-1,84)$ & $1,76(1,50-2,17)$ & 0,019 \\
\hline FGe por cistatina $\left(\mathrm{mL} / \mathrm{min} / 1,73 \mathrm{~m}^{2}\right)$ & $47,3(34,8-66,4)$ & $47,7(31,5-64,3)$ & $45,5(33,0-57,8)$ & $35,2(26,6-43,5)$ & 0,019 \\
\hline Urea al ingreso $(\mathrm{g} / \mathrm{dL})$ & $0,60(0,42-0,90)$ & $0,50(0,39-0,70)$ & $0,62(0,44-0,82)$ & $0,67(0,50-1,08)$ & 0,021 \\
\hline Creatinina al ingreso $(\mathrm{mg} / \mathrm{dL})$ & $1,17(0,96-1,39)$ & $1,03(0,84-1,40)$ & $1,16(0,88-1,56)$ & $1,31(1,09-1,75)$ & 0,014 \\
\hline \multicolumn{6}{|l|}{ Parámetros analíticos al ingreso } \\
\hline Ácido úrico (mg/dL) & $7,73(1,34)$ & $7,90(2,67)$ & $8,07(2,30)$ & $8,87(2,90)$ & 0,211 \\
\hline Sodio (mmol/L) & $143(140-144)$ & $142(140-143)$ & $141(139-144)$ & $139(136-143)$ & 0,015 \\
\hline Potasio ( $\mathrm{mmol} / \mathrm{L})$ & $4,00(0,59)$ & $4,00(0,50)$ & $4,09(0,55)$ & $4,06(0,69)$ & 0,700 \\
\hline Proteínas totales $(\mathrm{g} / \mathrm{L})$ & $7,00(0,63)$ & $6,66(0,51)$ & $6,27(0,60)$ & $6,06(0,94)$ & $<0,001$ \\
\hline Albúmina $(\mathrm{g} / \mathrm{dL})$ & $3,7(3,5-3,8)$ & $3,3(3,2-3,5)$ & $3,0(2,7-3,3)$ & $2,5(2,3-2,8)$ & $<0,001$ \\
\hline Colesterol total $(\mathrm{mg} / \mathrm{dL})$ & $178(154-185)$ & $147(131-182)$ & $137(115-159)$ & $114(99-134)$ & $<0,001$ \\
\hline Triglicéridos (mg/dL) & $128(86-172)$ & $96(80-126)$ & $87(73-107)$ & $80(63-92)$ & $<0,001$ \\
\hline Hemoglobina $(\mathrm{g} / \mathrm{L})$ & $13,1(1,9)$ & $13,0(1,8)$ & $11,6(1,9)$ & $11,2(1,9)$ & $<0,001$ \\
\hline Hematocrito (\%) & $40,5(5,8)$ & $39,3(5,7)$ & $35,4(6,0)$ & $34,5(6,0)$ & $<0,001$ \\
\hline $\operatorname{ADE}(\%)$ & $16,0(13,9-17,5)$ & $15,3(14,4-16,5)$ & $16,0(14,7-17,9)$ & $15,6(14,4-16,9)$ & 0,077 \\
\hline Linfocitos $\left(\mathrm{mm}^{3} / \mathrm{mL}\right)$ & $1.800(1.600-1.950)$ & $1.500(1.100-2.000)$ & $900(600-1.200)$ & $700(500-850)$ & $<0,001$ \\
\hline
\end{tabular}

Cómo citar este artículo: Rubio-Gracia J, et al. Valor pronóstico de la desnutrición en pacientes con insuficiencia cardíaca aguda y su influencia en la interpretación de marcadores de congestión venosa sistémica. Med Clin (Barc). 2020. https://doi.org/10.1016/j.medcli.2020.06.066 
Tabla 1 (continuación)

\begin{tabular}{|c|c|c|c|c|c|}
\hline Variables & Normal & Leve & Moderado & Severo & p-valor \\
\hline IST (\%) & $16,6(8,4-22,6)$ & $12,9(9,0-23,1)$ & $12,0(8,7-21,1)$ & $15,6(9,6-23,9)$ & 0,817 \\
\hline Ferritina (ng/mL) & $67,5(32,0-141,5)$ & $80,0(41,0-152,5)$ & $85,0(50,0-170,7)$ & $193,0(120,0-350,7)$ & 0,001 \\
\hline $\begin{array}{l}\text { Análisis del estado nutricional } \\
\text { Índice PNI (puntuación total) }\end{array}$ & $46,5(44,3-48,8)$ & $41,5(39,7-43,4)$ & $35,5(32,5-37,0)$ & $28,9(27,7-31,0)$ & $<0,001$ \\
\hline \multicolumn{6}{|l|}{ Objetivo primario ( $n[\%])$} \\
\hline Muerte para todas las causas a un año & $3(23,1)$ & $14(14,0)$ & $49(26,8)$ & $19(51,4)$ & $<0,001$ \\
\hline Muerte cardiovascular al año & $3(23,1)$ & $11(11,5)$ & $41(21,9)$ & $15(41,7)$ & 0,002 \\
\hline
\end{tabular}

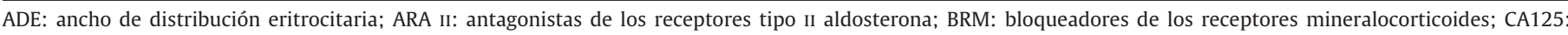

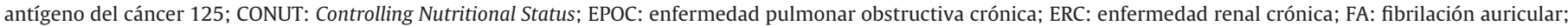

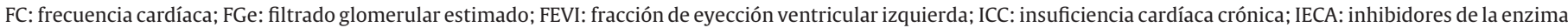

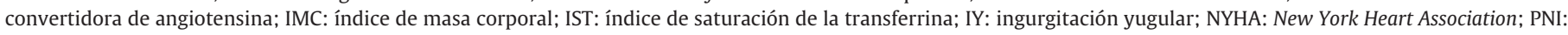
Prognostic Nutritional Index; TAD: tensión arterial diastólica; TAS: tensión arterial sistólica; VCI: vena cava inferior; VPr: volumen plasmático relativo.

partir de las concentraciones al ingreso de albúmina sérica (g/L), colesterol total $(\mathrm{mg} / \mathrm{dL})$ y linfocitos totales $\left(\mathrm{mm}^{3}\right)$. Los pacientes se clasificaron según la puntuación total según el siguiente esquema, de 0 a 1 estado nutricional normal; 2 a 4: desnutrición leve; 5 a 8 : desnutrición moderada y 9 a 12: desnutrición grave. 2) Índice de $\mathrm{PNI}^{17}$, calculado según la siguiente fórmula: [10 $\mathrm{x}$ albúmina sérica al ingreso $(\mathrm{g} / \mathrm{dL})+0,005 \mathrm{x}$ linfocitos totales $\left(\mathrm{mm}^{3}\right)$ al ingreso]. Los pacientes se clasificaron con base en la puntuación total del índice de PNI de la siguiente manera: > 38: estado nutricional normal; 35 a 38: desnutrición moderada $\mathrm{y}<35$ : desnutrición severa.

\section{Marcadores de congestión}

La analítica sanguínea al ingreso fue realizada por el laboratorio del hospital siguiendo procedimientos convencionales. La fracción aminoterminal del péptido natriurético cerebral (NT-proBNP $[\mathrm{pg} / \mathrm{mL}]$ ) (Modular Analytics Analyzer E601 Roche Diagnostics ${ }^{\circledR}$ $\mathrm{GmbH}$, Mannheim, Germany) y el antígeno carbohidrato 125 (CA125, U/mL) (Roche Diagnostics ${ }^{\circledR} \mathrm{GmbH}$, Mannheim, Germany), fueron seleccionados como biomarcadores de estrés ventricular y congestión respectivamente, siendo analizados durante las primeras 24 horas de ingreso y al alta. La cistatina $\mathrm{C}(\mathrm{mg} / \mathrm{L})$ (Latex N Test, con BN II Dade Behring GmbH ${ }^{\circledR}$, Marburg, Germany) se utilizó como biomarcador de función renal.

El volumen plasmático relativo (rPVS) se calculó como: ([volumen plasmático actual - volumen plasmático ideal]/volumen ideal plasmático) x 100. El volumen plasmático actual se estimó mediante el peso $(\mathrm{kg})$ y el hematocrito (\%) al ingreso de la siguiente manera: (1 - hematocrito) x ( $\mathrm{a}+$ [b x peso]), siendo «a" $\mathrm{y}$ «b» constantes con un valor de 1.530 para varones y 864 para mujeres y 41 para varones y 39 para mujeres, respectivamente ${ }^{18}$. El volumen plasmático ideal se calculó como: c x peso, donde "c» es una constante con un valor de 39 para los varones y 40 para las mujeres.

La presencia de "líneas b» pulmonares se midió al ingreso mediante los ecógrafos V-Scan y LOGIC F6 (General Electrics Healthcare ${ }^{\circledR}$ ), dividiendo cada hemitórax del paciente en cuatro cuadrantes (en total se exploraron ocho cuadrantes). Se consideró como significativa, aquella exploración con tres o más «líneas b» de manera bilateral. El diámetro de vena cava inferior (VCI) se estimó de manera visual como "dilatada» cuando alcanzaba una longitud de $20 \mathrm{~mm}$ o más medida a $2 \mathrm{~cm}$ de la entrada en la aurícula derecha, y se estimó que no existía un colapso inspiratorio si en inspiración forzada el diámetro se reducía menos del $50 \%$.

\section{Objetivo primario}

El objetivo primario del estudio fue la muerte por cualquier causa al año, iniciado desde el alta del paciente tras el ingreso indice por un episodio de IC descompensada. Dicha variable fue registrada por un investigador independiente mediante la revisión de las historias clínicas.

\section{Análisis estadístico}

Las variables cuantitativas se expresaron mediante la media \pm desviación standard o mediana (percentil 25-percentil 75), según siguieran la normalidad tras ser valorada mediante el test de Kolmogorov-Smirnoff. Para el contraste de hipótesis, se emplearon los test de la t de Student y ANOVA para las variables paramétricas y U de Mann-Whitney y Kruskal Wallis para las variables no paramétricas. Las variables categóricas se analizaron mediante el test de $\chi^{2}$ o test de Pearson, en el caso de que el valor esperado para ambos grupos fuese inferior a cinco. El estudio de correlación entre variables cuantitativas continuas se realizó mediante el test $\mathrm{R}^{2}$ de Pearson $\mathrm{y} / \mathrm{o} \rho$ de Spearman, según la distribución de la muestra. No se realizaron imputaciones por las pérdidas dado que se seleccionaron únicamente aquellos pacientes con todas las variables a estudio registradas en la base de datos.

El tiempo hasta el evento se representó mediante las curvas de supervivencia de Kaplan-Meier, usando el test de log-rank para realizar las comparaciones entre los diferentes grupos. La relación entre las escalas de nutrición estudiadas (CONUT y PNI) y el objetivo primario de muerte por todas las causas al año, se analizó mediante el análisis de regresión de Cox. Para el análisis multivariante, se seleccionaron aquellas variables con una $\mathrm{p}<20 \%$ en el análisis univariante de Cox y que no fueran variables empleadas para el cálculo de los índices de CONUT y PNI. Se realizó trasnformación mediante logaritmos fraccionados y tipificación en aquellas variables que no seguían la normalidad. El método elegido para el diseño del análisis multivariante fue el "análisis condicional hacia atrás».

Las variables se muestran con una estimación aproximada del $95 \%$ con sus respectivos intervalos de confianza. Un valor $\mathrm{p}<0,05$ se consideró estadísticamente significativo. Para el análisis se utilizaron los programas estadísticos SPSS versión 24.0. (IBM Corp. ${ }^{\circledR}$, Armonk, NY) y Jamovi 1.2.2.

\section{Resultados}

\section{Características basales de la muestra}

Desde enero de 2013 a diciembre de 2018, se incluyeron un total de 388 pacientes. Tras el análisis preliminar, 309 pacientes fueron incluidos en el análisis final (Figura suplementaria 1). La mediana de edad se situó en 82 años, con una proporción similar de hombres y mujeres (49,5\% vs. 50,5\%). La prevalencia de IC crónica fue cercana al $70 \%$. Los pacientes presentaban una alta carga de comorbilidades, siendo las más frecuentes la hipertensión arterial (83,8\%), la fibrilación/flutter auricular (58,9\%), la dislipemia $(45,8 \%)$ o la diabetes $(40,8 \%)$. Aproximadamente un tercio de los 
Tabla 2

Características basales según el índice PNI

\begin{tabular}{|c|c|c|c|c|}
\hline Variables & Normal & Moderado & Severo & p-valor \\
\hline$N(\%)$ & $117(37,9)$ & $83(26,9)$ & $109(35,3)$ & \\
\hline Edad (años) & $80,0(71,7-84,4)$ & $83,0(80,0-86,0)$ & $84,0(79,1-88,0)$ & $<0,001$ \\
\hline Género: (hombres[\%]) & $56(47,9)$ & $38(45,8)$ & $59(54,1)$ & 0,468 \\
\hline Peso $(k g)$ & $77,0(65,2-90,0)$ & $72,2(59,9-80,0)$ & $74,0(65,5-83,0)$ & 0,067 \\
\hline Altura $(m)$ & $1,59(1,53-1,67)$ & $1,60(1,53-1,68)$ & $1,60(1,50-1,68)$ & 0,643 \\
\hline $\operatorname{IMC}\left(\mathrm{kg} / \mathrm{m}^{2}\right)$ & $30,2(25,9-34,3)$ & $27,5(24,6-30,8)$ & $28,0(25,3-33,9)$ & 0,031 \\
\hline TAS $(\mathrm{mmHg})$ & $135(124-156)$ & $140(120-159)$ & $130(113-149)$ & 0,025 \\
\hline $\mathrm{TAD}(\mathrm{mmHg})$ & $75(65-86)$ & $74(64-85)$ & $69(59-77)$ & 0,001 \\
\hline$F C(l / p / m)$ & $77(66-93)$ & $80(67-96)$ & $78(68-92)$ & 0,861 \\
\hline \multicolumn{5}{|l|}{ Signos de IC (n[\%]) } \\
\hline IY & $66(57,4)$ & $54(67,5)$ & $68(64,2)$ & 0,324 \\
\hline Edemas & $94(81,7)$ & $64(80,0)$ & $84(78,5)$ & 0,833 \\
\hline Crepitantes & $68(70,1)$ & $50(72,5)$ & $68(73,9)$ & 0,841 \\
\hline \multicolumn{5}{|l|}{ Parámetros ecocardiográficos } \\
\hline FEVI (\%) & $50,0(39,0-62,0)$ & $54,5(43,0-63,1)$ & $59,0(44,2-65,0)$ & 0,040 \\
\hline $\mathrm{PAP}(\mathrm{mmHg})$ & $42(30-60)$ & $51(40-59)$ & $44(35-55)$ & 0,561 \\
\hline Diámetro $\mathrm{AI}(\mathrm{mm})$ & $48,3(9,4)$ & $48,8(8,9)$ & $48,6(9,4)$ & 0,940 \\
\hline Masa ventricular (g) & $127(42)$ & $124(59)$ & $117(43)$ & 0,508 \\
\hline «Líneas b» ecográficas (presencia) & $40(44,9)$ & $38(62,3)$ & $51(70,8)$ & 0,003 \\
\hline Dilatación VCI ( $\geq 50 \%)$ & $19(39,6)$ & $17(54,8)$ & $22(59,5)$ & 0,157 \\
\hline Clase funcional basal NYHA (n[\%]) & & & & 0,458 \\
\hline I & $17(14,7)$ & $7(8,9)$ & $9(8,7)$ & \\
\hline II & $71(61,2)$ & $54(68,4)$ & $63(60,6)$ & \\
\hline III & $28(24,1)$ & $18(22,8)$ & $31(29,8)$ & \\
\hline IV & $0(0,0)$ & $0(0,0)$ & $1(1,0)$ & \\
\hline \multicolumn{5}{|l|}{ Antecedentes ( $n[\%])$} \\
\hline ICC & $83(70,9)$ & $54(65,9)$ & $77(70,6)$ & 0,706 \\
\hline Hipertensión & $98(83,8)$ & $66(79,5)$ & $95(87,2)$ & 0,363 \\
\hline Cardiopatía isquémica & $44(37,6)$ & $22(26,5)$ & $31(28,4)$ & 0,177 \\
\hline Dislipemia & $64(54,7)$ & $32(39,0)$ & $45(41,3)$ & 0,046 \\
\hline Diabetes & $47(40,2)$ & $35(42,2)$ & $44(40,4)$ & 0,955 \\
\hline FA/flutter & $69(59,0)$ & $46(55,4)$ & $67(61,5)$ & 0,700 \\
\hline EPOC & $17(14,5)$ & $15(18,1)$ & $18(16,5)$ & 0,793 \\
\hline ERC & $32(27,4)$ & $26(31,7)$ & $40(36,7)$ & 0,321 \\
\hline Anemia & $22(19,0)$ & $18(21,7)$ & $37(34,3)$ & 0,022 \\
\hline Portador marcapasos & $14(12,0)$ & $10(12,2)$ & $9(8,3)$ & 0,586 \\
\hline \multicolumn{5}{|l|}{ Tratamiento IC ( $n[\%])$} \\
\hline Diuréticos de asa & $104(89,7)$ & $72(90,0)$ & $90(89,1)$ & 0,980 \\
\hline Betabloqueadores & $68(58,6)$ & $38(47,5)$ & $51(50,5)$ & 0,260 \\
\hline IECA/ARA II & $90(77,6)$ & $60(75,0)$ & $64(63,4)$ & 0,053 \\
\hline BRM & $45(38,8)$ & $12(15,2)$ & $30(30,0)$ & 0,002 \\
\hline Variables & Normal & Moderado & Severo & p-valor \\
\hline \multicolumn{5}{|l|}{ Biomarcadores } \\
\hline NT-proBNP al ingreso (pg/mL) & $2.523(1.597-5.317)$ & $3.989(1.411-7.632)$ & $4.640(2.595-8.982)$ & $<0,001$ \\
\hline NT-proBNP al alta $(\mathrm{pg} / \mathrm{mL})$ & $1.967(794-3.755)$ & $2.127(810-4.154)$ & $3.432(1.618-5.109)$ & 0,004 \\
\hline CA125 al ingreso (mU/mL) & $52,5(20,4-131,0)$ & $48,5(20,3-112,8)$ & $58,1(35,7-107,6)$ & 0,458 \\
\hline CA125 al alta $(\mathrm{mU} / \mathrm{mL})$ & $43,4(15,3-134,6)$ & $58,9(21,3-116,8)$ & $83,0(35,9-150,9)$ & 0,066 \\
\hline \multicolumn{5}{|l|}{ Análisis indirecto del volumen plasmático } \\
\hline Volumen plasmático real (mL) & $2.854(2.464-3.146)$ & $2.712(2.364-3.083)$ & $2.970(2.682-3.262)$ & 0,041 \\
\hline Volumen plasmático ideal (mL) & $3.023(2.600-3.568)$ & $2.817(2.398-3.123)$ & $2.886(2.593-3.237)$ & 0,068 \\
\hline $\operatorname{VPr}(\%)$ & $-6,3([-12,5]-[0,5])$ & $-2,6([-10,2]-[4,6])$ & $-0,9([-6,8]-[8,1])$ & 0,001 \\
\hline \multicolumn{5}{|l|}{ Parámetros de función renal } \\
\hline Cistatina al ingreso $(\mathrm{mg} / \mathrm{mL})$ & $1,41(1,12-1,93)$ & $1,42(1,20-1,87)$ & $1,55(1,30-2,00)$ & 0,156 \\
\hline FGe por cistatina $\left(\mathrm{mL} / \mathrm{min} / 1,73 \mathrm{~m}^{2}\right)$ & $47,1(30,9-63,7)$ & $46,8(32,4-58,2)$ & $41,7(29,5-52,6)$ & 0,156 \\
\hline Urea al ingreso (g/dL) & $0,51(0,40-0,75)$ & $0,64(0,48-0,82)$ & $0,62(0,45-0,81)$ & 0,072 \\
\hline Creatinina al ingreso (mg/dL) & $1,09(0,88-1,43)$ & $1,17(0,86-1,57)$ & $1,15(0,92-1,59)$ & 0,591 \\
\hline \multicolumn{5}{|l|}{ Parámetros analíticos al ingreso } \\
\hline Ácido úrico (mg/dL) & $8,0(2,4)$ & $7,9(2,6)$ & $8,3(2,3)$ & 0,406 \\
\hline Sodio (mmol/L) & $142(140-144)$ & $141(139-143)$ & $141(138-143)$ & 0,188 \\
\hline Potasio $(\mathrm{mmol} / \mathrm{L})$ & $4,04(0,52)$ & $4,08(0,53)$ & $4,05(0,62)$ & 0,854 \\
\hline Proteínas totales $(\mathrm{g} / \mathrm{L}$ ) & $6,65(0,52)$ & $6,42(0,49)$ & $6,09(0,80)$ & $<0,001$ \\
\hline Albúmina $(\mathrm{g} / \mathrm{dL})$ & $3,4(3,2-3,6)$ & $3,2(3,0-3,3)$ & $2,7(2,5-2,8)$ & $<0,001$ \\
\hline Colesterol total $(\mathrm{mg} / \mathrm{dL})$ & $142(117-169)$ & $142(123-162)$ & $134(111-155)$ & 0,064 \\
\hline Triglicéridos (mg/dL) & $93(80-125)$ & $87(76-103)$ & $84(68-116)$ & 0,001 \\
\hline Hemoglobina $(\mathrm{g} / \mathrm{L})$ & $12,6(1,9)$ & $12,1(1,9)$ & $11,2(1,8)$ & $<0,001$ \\
\hline Hematocrito (\%) & $38,6(5,8)$ & $37,2(5,7)$ & $34,1(6,2)$ & $<0,001$ \\
\hline $\operatorname{ADE}(\%)$ & $15,5(14,4-16,8)$ & $15,8(14,8-17,5)$ & $15,8(14,7-17,7)$ & 0,360 \\
\hline Linfocitos $\left(\mathrm{mm}^{3} / \mathrm{mL}\right)$ & $1.500(1.100-2.000)$ & $900(600-1.200)$ & $700(500-1.000)$ & $<0,001$ \\
\hline
\end{tabular}

Cómo citar este artículo: Rubio-Gracia J, et al. Valor pronóstico de la desnutrición en pacientes con insuficiencia cardíaca aguda y su influencia en la interpretación de marcadores de congestión venosa sistémica. Med Clin (Barc). 2020. https://doi.org/10.1016/j.medcli.2020.06.066 
Tabla 2 (continuación)

\begin{tabular}{|c|c|c|c|c|}
\hline Variables & Normal & Moderado & Severo & p-valor \\
\hline IST (\%) & $12,8(9,0-22,4)$ & $11,5(8,0-21,7)$ & $15,9(9,1-24,2)$ & 0,413 \\
\hline Ferritina $(\mathrm{ng} / \mathrm{mL})$ & $64(35-139)$ & $85(42-116)$ & $156(77-309)$ & $<0,001$ \\
\hline $\begin{array}{l}\text { Análisis del estado nutricional } \\
\text { Îndice de CONUT (puntuación total) }\end{array}$ & $3(3-4)$ & $6(5-6)$ & $8(7-9)$ & $<0,001$ \\
\hline \multicolumn{5}{|l|}{ Objetivo primario ( $n[\%])$} \\
\hline Muerte para todas las causas a un año & $16(14,0)$ & $21(25,3)$ & $41(37,6)$ & $<0,001$ \\
\hline Muerte cardiovascular al año & $13(20,6)$ & $17(27,0)$ & $33(52,4)$ & 0,002 \\
\hline
\end{tabular}

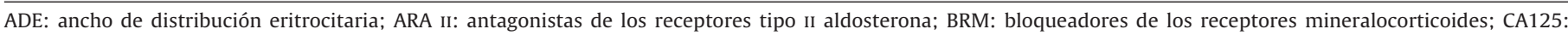

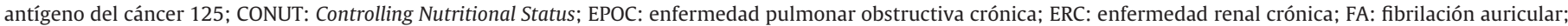

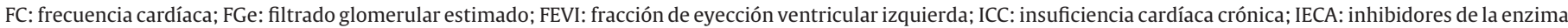

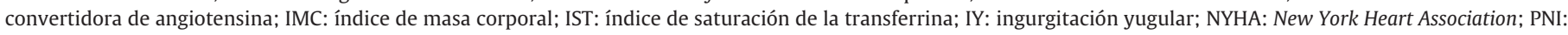
Prognostic Nutritional Index; TAD: tensión arterial diastólica; TAS: tensión arterial sistólica; VCI: vena cava inferior; VPr: volumen plasmático relativo.

pacientes presentaban antecedentes de enfermedad renal crónica (filtrado glomerular estimado (FGe) calculado por CKD-EPI creatinina $<60 \mathrm{~mL} / \mathrm{min} / 1,72 \mathrm{~m}^{3}$ ).

La mediana de NT-proBNP al ingreso fue de $3.400 \mathrm{pg} / \mathrm{mL}$ (1.915$7.151)$ y la de CA125 al ingreso de $55,2 \mathrm{U} / \mathrm{mL}(23,8-116,8)$. La mediana de volumen plasmático relativo (VPr) fue de $-3,1 \%(-11,0-$ $3,7)$.

Un análisis completo de las características basales de la población incluida puede consultarse en la Tabla suplementaria 1.

\section{Análisis estratificado según el estado nutricional al ingreso (tablas 1 y 2)}

Elanálisis de la muestra mostró una alta prevalencia de desnutrición en los pacientes incluidos. Según la escala CONUT, la gran mayoría de los pacientes presentaban desnutrición: un 28,8\% ligera, el $55 \%$ moderada y un $12 \%$ severa. La escala PNI identificó una menor proporción de pacientes con desnutrición: un 35,3\% moderada y un $26,9 \%$ severa; aunque hay que considerar que ambas escalas difieren ligeramente (el PNI no posee una categoría de desnutrición ligera). La correlación entre ambas escalas de nutrición fue muy alta ( $\rho$ de Spearman $=0,897 ; \mathrm{p}=<0,001$ ) (tablas 1 y 2 ).

En comparación con aquellos pacientes sin desnutrición, los pacientes con algún grado de desnutrición, ya fuera medida mediante la escala CONUT o PNI, presentaban al ingreso más edad, cifras de tensión arterial sistólica (TAS) y diastólica (TAD) más bajas, una mayor proporción de «líneas b» ecográficas, mayor volumen plasmático relativo (VPr) y cifras más bajas de hemoglobina y hematocrito. La concentración de NT-proBNP, tanto al ingreso como al alta, también fueron mayores en los pacientes con algún grado de desnutrición.

Además, los pacientes con desnutrición, según la escala CONUT, presentaban una mayor proporción de ingurgitación yugular (IY) $(\mathrm{p}=0,020)$ al ingreso, y contaban en mayor proporción con antecedentes previos de cardiopatía isquémica (ICD) $(p=0,013)$ y enfermedad renal crónica (ERC) $(\mathrm{p}=0,037)$. Al alta, también presentaron mayores concentraciones de CA125 ( $p=0,022)$.

Según la escala PNI, los pacientes malnutridos tenían un menor índice de masa corporal (IMC) $(\mathrm{p}=0,031)$, mayor fracción de eyección ventricular izquierda (FEVI) $(\mathrm{p}=0,040)$, mayor proporción de dislipemia $(p=0,046)$ y anemia $(p=0,022)$ previas al ingreso. La prescripción de bloqueadores de los mineralocorticoides (BRM) fue menor entre los desnutridos $(\mathrm{p}=0,002)$.

\section{Análisis de la mortalidad según las escalas de desnutrición}

Durante el año posterior al ingreso, se produjeron un total 91 fallecimientos por todas las causas (26\%), 76 pacientes fallecieron por enfermedades cardiovasculares (83,5\%); en la
Tabla suplementaria 3 puede consultarse la distribución según la causa fundamental de la muerte en cada sujeto.

La distribución de dichas muertes según la escala CONUT fue de un $23,1 \%$ en el grupo «normal», $14 \%$ en el grupo «ligera», $26,8 \%$ en "moderada" y de un 51,4\% en el grupo de pacientes con desnutrición "severa». Para la escala PNI, la distribución de eventos fue de un $14 \%$ para el grupo "normal», $26,6 \%$ en el grupo "moderado» y de un $52,6 \%$ para el grupo con desnutrición «severa». Las curvas de supervivencia se muestran en las figuras 1 y 2, siendo las diferencias para el objetivo de muerte para todas las causas superior de manera significativa en el grupo de pacientes con mayor grado de desnutrición, $\mathrm{p}=<0,001$ para el CONUT y $\mathrm{p}=<0,001$ para el PNI respectivamente.

El análisis univariante de Cox para el objetivo primario de muerte por cualquier causa, identificó la escala de valoración nutricional de CONUT (HR 1,62 [1,22-2,14]; p = 0,001) y de PNI (HR 0,65 [0,53-0,80]; $\mathrm{p}=<0,001$ ) como factores asociados de manera significativa a la mortalidad al año por todas las causas.

En el análisis multivariante, realizado tras ajustar por 20 variables diferentes asociadas al objetivo primario, la significación de las escalas nutricionales desapareció en detrimento de variables directas como las concentraciones de ácido úrico (HR 1,24 [1,07-1,45]; $\mathrm{p}=0,006)$, el hematocrito (HR 0,90 [0,83-0,99]; $\mathrm{p}=0,023)$ o la ferritina, ambas al ingreso (HR 1,89 [1,19-3]; p = 0,007). El análisis de regresión univariante y multivariante de Cox completo puede ser consultado en la Tabla suplementaria 2.

Dichos resultados fueron similares cuando se analizaron las escalas nutricionales respecto al objetivo de muerte cardiovascular (Tabla suplementaria 4).

\section{Discusión}

La desnutrición es una comorbilidad asociada a la IC con una frecuencia variable según la fuente consultada ${ }^{3}$. Este hecho se debe fundamentalmente a las diferentes herramientas empleadas para su detección durante un episodio de ICA descompensada. En nuestra muestra, ambas escalas, la de CONUT y PNI, identificaron una alta proporción de desnutrición entre los pacientes con IC ingresados por descompensación, siendo la prevalencia superior a la descrita en otras series ${ }^{8}$.

Estos resultados pueden ser debidos a la mayor edad media y la carga de comorbilidades propia de los pacientes en Medicina Interna $^{2}$; la mayoría de los estudios descriptivos están basados en cohortes de pacientes procedentes de los servicios de Cardiología, donde existe una mayor prevalencia de IC de origen isquémico, con FEVI reducida ${ }^{19}$. Además, las variables empleadas para el cálculo de los índices de nutrición, pueden verse influenciadas por el tratamiento habitual de los pacientes, como las estatinas, ya que normalmente son pacientes que presentan un muy alto riesgo cardiovascular, no solo coronario ${ }^{20}$, que obliga a ser más exigente con 


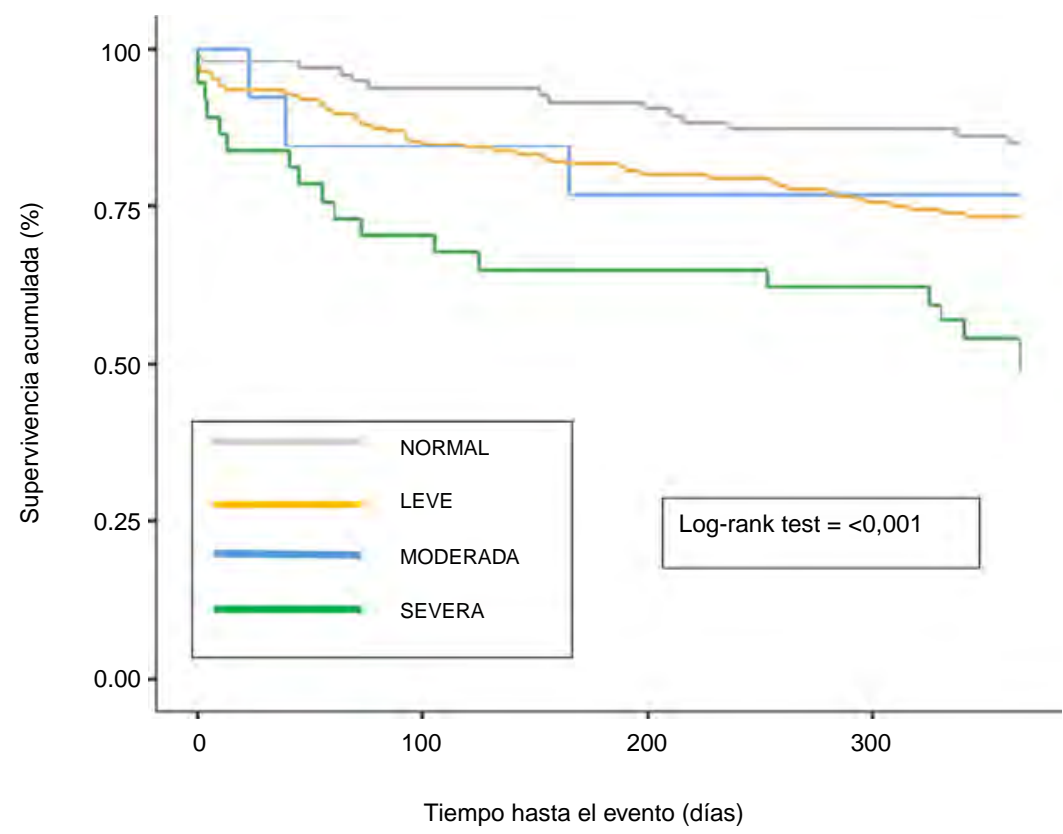

Figura 1. Curvas de supervivencia para el objetivo primario de muerte por todas las causas al año de ingreso según el índice CONUT.

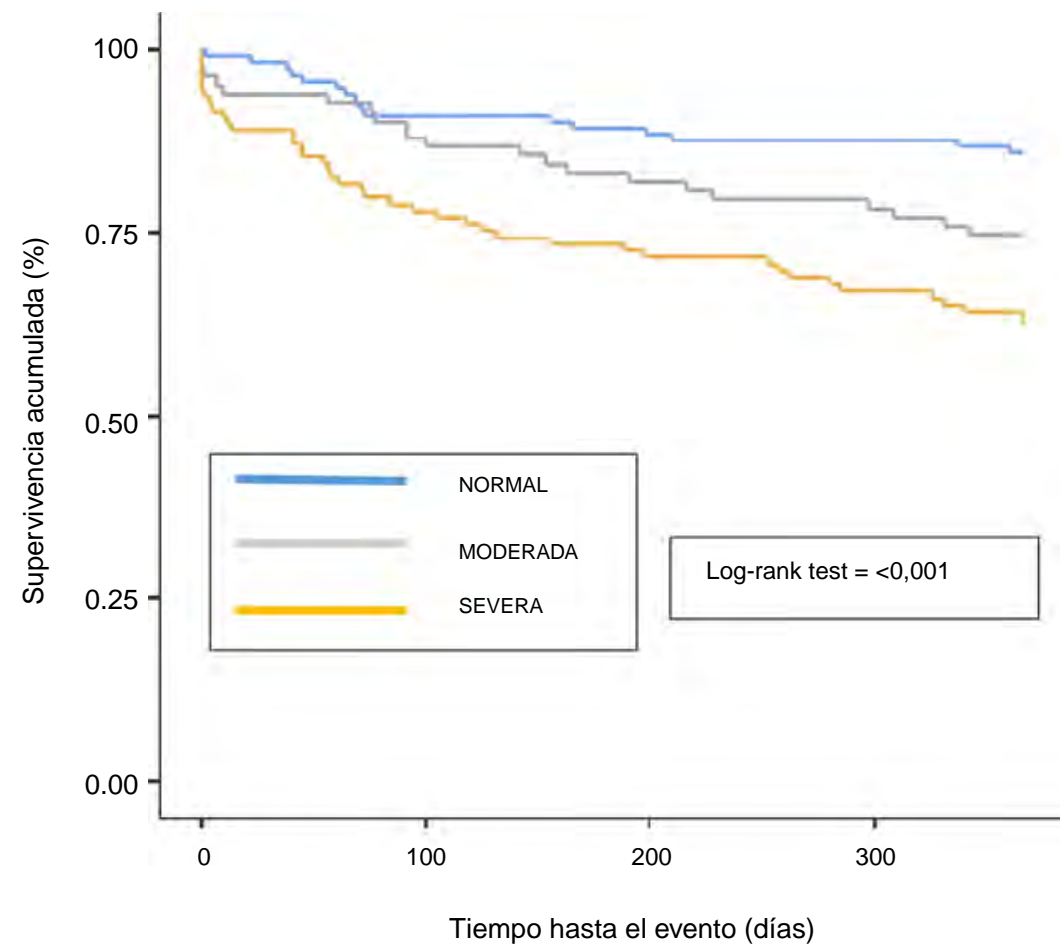

Figura 2. Curvas de supervivencia para el objetivo primario de muerte por todas las causas tras el ingreso según el índice PNI.

los objetivos de reducción de colesterol. Por lo tanto, la escala de CONUT ha de ser interpretada con cautela en este perfil de pacientes, dado que podría sobreestimar el grado de desnutrición ${ }^{6}$. En nuestro análisis, no se recogieron las dosis totales de estatinas por paciente, lo que podría suponer un falso incremento del grado de desnutrición según la escala CONUT y una limitación a la hora de extrapolar los resultados. En este sentido, la implementación de métodos como el análisis bioimpedanciométrico (BiVA) ${ }^{21,22}$, podría aportar un valor añadido, tanto para el cálculo de la congestión, como del estado nutricional de los pacientes.
El grado de congestión sistémica de los pacientes de este estudio, ya fuese medida mediante parámetros analíticos (NT-proBNPo CA125), por el uso de la ecografía pulmonar («líneas b») o mediante el cálculo indirecto del volumen plasmático, fue superior en los grupos de pacientes que presentaban un mayor grado de desnutrición, tanto para la escala de CONUT como para la PNI. En la IC hay una tendencia a la expansión del volumen extracelular propiciada por la respuesta neuroendocrina a la hipoperfusión tisular en la ICFEr, o el estado inflamatorio propio de la ICFEp, que se manifiesta a través de los signos propios de la congestión ${ }^{11,23}$. A su vez, la

Cómo citar este artículo: Rubio-Gracia J, et al. Valor pronóstico de la desnutrición en pacientes con insuficiencia cardíaca aguda y su influencia en la interpretación de marcadores de congestión venosa sistémica. Med Clin (Barc). 2020. https://doi.org/10.1016/j.medcli.2020.06.066 
desnutrición puede acentuar la clínica congestiva, por la disminución de la presión oncótica del plasma, secundaria a la hipoalbuminemia ${ }^{24}$. Para cerrar este círculo vicioso, la congestión puede dificultar una correcta identificación del grado de desnutrición. Así, por ejemplo, la expansión de volumen junto a la sarcopenia puede inducir a error en la estimación del filtrado glomerular por creatinina, o al atribuir la hipoalbuminemia, la anemia o los edemas exclusivamente a la congestión sistémica, sin tener en cuenta factores inflamatorios o nutricionales ${ }^{3}$.

Además de razones clínicas, existen mecanismos fisiopatológicos que explican, al menos en parte, la compleja relación entre congestión y desnutrición. La activación de vías fisiopatológicas complejas $^{3}$, resultado de la disfunción ventricular izquierda crónica y la readaptación de la homeostasis mediante el sistema renina angiotensina aldosterona y el sistema nervioso simpático. El estímulo prolongado de ambas vías, es capaz de propiciar un incremento de la resistencia insulínica, situación que disminuye la glucólisis y la obtención de ATP a nivel del miocardiocito, disminuyendo la actividad de la cadena respiratoria a nivel mitocondrial ${ }^{24}$. Esta situación se traduce en última instancia, en una disminución de la contracción del ventrículo izquierdo, agravando la disfunción ventricular izquierda e incrementando la congestión venosa sistémica ${ }^{25}$. Por otro lado, una alta proporción de los pacientes con IC crónica también presentan ERC ${ }^{26}$, situación que se ve agravada al empeorar el gasto cardíaco y que contribuye a prolongar la situación de "caquexia" que los pacientes presentan en estadios avanzados del síndrome cardiorrenal ${ }^{27}$. Esto explicaría porque en nuestros resultados los pacientes con mayor desnutrición mostraron mayores grados de anemia, a pesar de unos niveles más elevados de ferritina en sangre, situación compatible con un estado pro-inflamatorio sostenido ${ }^{3}$.

La desnutrición medida a través de escalas y parámetros analíticos ha demostrado ser un marcador de riesgo de muerte y reingresos por $\mathrm{IC}^{4,5}$, si bien es cierto que dicha situación clínica pierde potencia estadística en los diferentes análisis multivariantes desarrollados al comparar las escalas de nutrición frente a otras variables en los pacientes con IC, como la función renal o los péptidos natriuréticos ${ }^{8}$.

En nuestro estudio, obtuvimos resultados similares a los encontrados en la literatura; la desnutrición, ya fuese medida por el CONUT o el PNI, incrementó el riesgo de muerte por cualquier causa al año del alta en el análisis univariante. Sin embargo, al introducir dichas variables en el análisis multivariante, perdieron la significación estadística a favor de otras, como las concentraciones de ácido úrico, el hematocrito o la ferritina.

En nuestra experiencia, la desnutrición, ya fuese cuantificada por la escala de CONUT o el PNI, es muy prevalente en los pacientes ingresados por IC descompensada. Los diferentes grados de nutrición, independientemente de la herramienta empleada (CONUT o $\mathrm{PNI}$ ), se asociaron de manera significativa con concentraciones más elevadas de NT-proBNP, CA125 y valores más altos de VPr, así como con una mayor detección de «líneas b pulmonares», por lo que la evaluación de la congestión en los pacientes con ICA a través de estos métodos, debería interpretarse con cautela en los pacientes desnutridos.

A raíz de los resultados de este estudio, podemos concluir que el empleo de escalas sencillas para valorar la desnutrición en pacientes ingresados por descompensación de la IC es factible y permite detectar una prevalencia alta de desnutrición. La desnutrición al ingreso se asocia con una mayor mortalidad por cualquier causa durante el seguimiento, quizás reflejo de un estado proinflamatorio sostenido. No obstante, un mayor grado de desnutrición puede contribuir a sobrevalorar el grado de congestión, por lo que deberían implementarse métodos más específicos en su evaluación que podrían conducir a una mejor estratificación del riesgo y una comprensión más precisa de la fisiopatología de las descompensaciones.

\section{Financiación}

Este proyecto no ha recibido ningún tipo de financiación.

\section{Conflicto de intereses}

Los autores declaran no tener ningún conflicto de intereses.

\section{Anexo. Material adicional}

Se puede consultar material adicional a este artículo en su versión electrónica disponible en https://doi.org/ 10.1016/j.medcli.2020.06.066.

\section{Bibliografía}

1. Ruiz-Laiglesia F-J, Sánchez-Marteles M, Pérez-Calvo J-I, Formiga F, BartoloméSatué JA, Armengou-Arxé A, et al. Comorbidity in heart failure. Results of the Spanish RICA registry. QJM. 2014;107:989-94.

2. Rahman A, Jafry S, Jeejeebhoy K, Nagpal AD, Pisani B, Agarwala R. Malnutrition and cachexia in heart failure. J PEN J Enteral Nutr. 2016;40:475-86.

3. Al-Najjar Y, Clark AL. Predicting outcome in patients with left ventricular systolic chronic heart failure using a nutritional risk index. Am J Cardiol. 2012;109:1315-20.

4. Lin $H$, Zhang $H$, Lin Z, Li X, Kong $X$, Sun G. Review of nutritional screening and assessment tools and clinical outcomes in heart failure. Heart Fail Rev. 2016;21:549-65.

5. Ignacio de Ulíbarri J, González-Madroño A, De Villar NGP, González P, González B, Mancha A, et al. CONUT: a tool for controlling nutritional status. First validation in a hospital population. Nutr Hosp. 2005;20:38-45.

6. Bouillanne O, Morineau G, Dupont C, Coulombel I, Vincent JP, Nicolis I, et al. Geriatric Nutritional Risk Index: a new index for evaluating at-risk elderly medical patients. Am J Clin Nutr. 2005;82:777-83.

7. Sze S, Pellicori P, Kazmi S, Rigby A, Cleland JGF, Wong K, et al. Prevalence and prognostic significance of malnutrition using 3 scoring systems among outpatients with heart failure: a comparison with body mass index. JACC Hear Fail. 2018;6:476-86

8. Rubio-Gracia J, Demissei BG, Ter Maaten JM, Cleland JG, O'Connor CM, Metra M, et al. Prevalence, predictors and clinical outcome of residual congestion in acute decompensated heart failure. Int J Cardiol. 2018;258:185-91.

9. Damy T, Kallvikbacka-Bennett A, Zhang J, Goode K, Buga L, Hobkirk J, et al. Does the physical examination still have a role in patients with suspected heart failure? Eur J Heart Fail. 2011;13:1340-8.

10. Miller WL, Mullan BP. Understanding the heterogeneity in volume overload and fluid distribution in decompensated heart failure is key to optimal volume management: role for blood volume quantitation. JACC Hear Fail. 2014;2:298-305.

11. Mullens W, Damman K, Harjola VP, Mebazaa A, Brunner-La Rocca HP, Martens P et al. The use of diuretics in heart failure with congestion - a position statement from the Heart Failure Association of the European Society of Cardiology. Eur J Heart Fail. 2019;21:137-55.

12. Lala A, McNulty SE, Mentz RJ, Dunlay SM, Vader JM, AbouEzzeddine OF, et al. Relief and recurrence of congestion during and after hospitalization for acute heart failure. Circ Hear Fail. 2015;8:741-8.

13. Núñez J, Llàcer $P$, Núñez E, Ventura $S$, Bonanad $C$, Bodí V, et al. Antigen carbohydrate 125 and creatinine on admission for prediction of renal function response following loop diuretic administration in acute heart failure. Int J Cardiol. 2014;174:516-23.

14. Ponikowski P, Voors AA, Anker SD, Bueno H, Cleland JGF, Coats AJS, et al. 2016 ESC Guidelines for the diagnosis and treatment of acute and chronic heart failure: The Task Force for the diagnosis and treatment of acute and chronic heart failure of the European Society of Cardiology (ESC). Developed with the special contribution of the Heart Failure Association (HFA) of the ESC. Eur J Heart Fail. 2016;18:891-975.

15. Lang RM, Badano LP, Mor-Avi V, Afilalo J, Armstrong A, Ernande L, et al. Recommendations for cardiac chamber quantification by echocardiography in adults an update from the American Society of Echocardiography and the European Association of Cardiovascular Imaging. J Am Soc Echocardiogr. 2015;28:1-39, e14.

16. Buzby GP, Mullen JL, Matthews DC, Hobbs CL, Rosato EF. Prognostic nutritional index in gastrointestinal surgery. Am J Surg. 1980;139:160-7.

17. Maznyczka AM, Barakat MF, Ussen B, Kaura A, Abu-Own H, Jouhra F, et al. Calculated plasma volume status and outcomes in patients undergoing coronary bypass graft surgery. Heart. 2019;105:1020-6.

18. Farmakis D, Parissis J, Lekakis J, Filippatos G. Acute heart failure: epidemiology risk factors, and prevention. Rev Esp Cardiol (Engl Ed.). 2015;68:245-8.

19. The Task Force Members, ESC Committee for Practical Guidelines, ESC Nationa Cardiac Societies. 2019 ESC /EAS Guidelines for the management of dyslipidaemias: lipid modification to reduce cardiovascular risk. Atherosclerosis. 2019;290:140-205

20. Di Somma S, Lukaski HC, Codognotto M, Peacock WF, Fiorini F, Aspromonte $\mathrm{N}$, et al. Consensus paper on the use of BIVA (Bioeletrical Impendance Vector 
Analysis) in medicine for the management of body hydration. Emerg Care J. 2013;7:6.

21. Pimenta J, Bettencourt P. A new tool to measure hydration status in acute heart failure - Is bioelectrical impedance vector analysis (BIVA) making its way to the wards? Rev Clin Esp. 2016;216:126-7.

22. Rubio Gracia J, Sánchez Marteles M, Pérez Calvo JI. Involvement of systemic venous congestion in heart failure. Rev Clin Esp. 2017;217:161-9.

23. Arques S. Does hypoalbuminemia play a role in the worsening of heart failure? Am J Cardiol. 2020;125:1755.
24. Saotome M, Ikoma T, Hasan P, Maekawa Y. Cardiac insulin resistance in heart failure: the role of mitochondrial dynamics. Int J Mol Sci. 2019;20:3552.

25. Hanna EB, Deschamps EH. Acute heart failure: acute cardiorenal syndrome and role of aggressive decongestion. Clin Cardiol. 2014:37:773-8.

26. Husain-Syed F, McCullough PA, Birk HW, Renker M, Brocca A, Seeger W, et al Cardio-pulmonary-renal interactions: a multidisciplinary approach. J Am Coll Cardiol. 2015;65:2433-48

27. Gnanaraj JF, Von Haehling S, Anker SD, Raj DS, Radhakrishnan J. The relevance of congestion in the cardio-renal syndrome. Kidney Int. 2013;83:384-91.

Cómo citar este artículo: Rubio-Gracia J, et al. Valor pronóstico de la desnutrición en pacientes con insuficiencia cardíaca aguda y su influencia en la interpretación de marcadores de congestión venosa sistémica. Med Clin (Barc). 2020. https://doi.org/10.1016/j.medcli.2020.06.066 